\title{
Embedded Modulable Self-Tuning Cavity for WDM-PON Transmitter
}

\author{
P. Parolari ${ }^{1}$, L. Marazzi ${ }^{1 *}$, M. Brunero ${ }^{1}$, P. Galli $^{2}$, G. Gavioli ${ }^{2}$, M. Martinelli ${ }^{1}$ \\ ${ }^{1}$ Dept. of Electronic and Information, Politecnico di Milano, Milano, 20133 Italy \\ *Tel: +3902 23998926, e-mail: marazzi@elet.polimi.it \\ ${ }^{2}$ Optics Division, Alcatel-Lucent Italia, Vimercate MB, 20871 Italy
}

\begin{abstract}
A network-embedded self-tuning cavity based on a self-seeded RSOA is analysed with particular regards to the impact of some parameters that characterize the transmitter. The cavity is defined by the SOA reflective mirror and a Faraday rotator mirror at the remote node, which allows polarization independent operation with low PDG RSOAs. The majority of the cavity is formed by the distribution fibre, while the arrayed waveguide grating (AWG) at the RN operates as the frequency selective element. The experimental analysis is carried out by varying fundamental cavity parameters such as the distribution fibre length, the cavity losses, the bandwidth of the AWG filter and its spectral location with respect to the RSOA gain peak. Performance is experimentally evaluated at $1.25 \mathrm{~Gb} / \mathrm{s}$. Transmitter output power is determined by cavity losses independently of the AWG bandwidth. On the other hand AWG bandwidth strongly influence BER performance: for lower FWHMs error floors appear associated to intensity noise.
\end{abstract}

Keywords: WDM PON, self-seeded RSOAs, performance analysis, colourless transmitter, embedded cavity.

\section{INTRODUCTION}

Wavelength Division Multiplexing passive optical networks (WDM PON) [1] have potential advantages for next-generation access networks (NGAN) allowing the full exploitation of the available bandwidth in the optical fibre. Essential is the adoption of the same optical network unit (ONU) for all users, which implies that the individual ONUs are "colourless", i.e., agnostic in wavelength. This result can be achieved by the optical injection into Fabry-Perot lasers [2] or in reflective semiconductor optical amplifiers (RSOAs) [3], exploiting a tuning source placed at the optical line terminal (OLT), which propagates in the same direction of the downstream signal (DS), but in another band [4], e.g. respectively in the L and C band. These solutions are impaired by the feeder-fiber distributed scattering of the tuning source, namely Rayleigh Back Scattering (RS) and the Brillouin Back Scattering [5]. The RS causes an incoherent crosstalk in the transmission of US signal, which limits the maximum bridged distance. A smart solution, which avoids the need of tuning external sources and therefore does not suffer from RS-related penalties, is represented by self-seeded RSOAs [6], which appear promising candidates to obtain directly-modulated self-tuning ONUs transmitters, with reduced costs compared to the externally-tuned RSOAs. Namely the transmitter is constituted by a cavity embedded in the network itself, in fact its two mirrors are located respectively at the ONU and at the RN and the majority of the cavity is formed by the distribution fibre, while the Arrayed waveguide grating (AWG) at the RN operates as the frequency selective element.

In this paper the performance of a network-embedded ONU transmitter based on a self-seeded RSOA are analyzed, with particular regards to the variation of some parameters that characterize the cavity such as: cavity length, cavity losses, bandwidth of the AWG filter and its spectral location with respect to the RSOA gain peak.

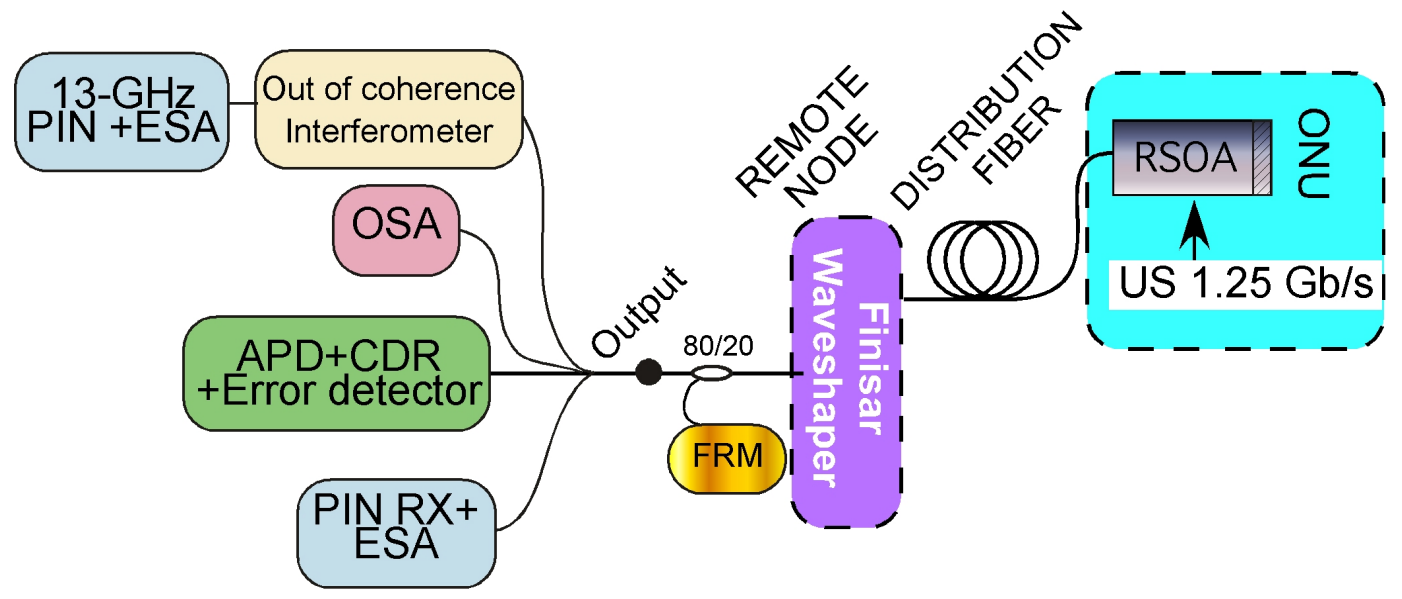

Figure 1. Experimental set up. 


\section{EXPERIMENTAL SET UP}

Figure 1 shows the experimental set up of the realized network-embedded ONU. The two cavity mirrors are evidenced: at the ONU, the reflecting mirror belonging to the RSOA and, at the remote node, the Faraday rotator mirror (FRM), which makes the configuration virtually polarization-independent with low polarization independent gain (PDG) RSOAs [7]. The output of the transmitter is taken at the RN via an 80/20 coupler. The AWG channel transfer function is implemented through a programmable filter (Finisar WaveShaper ${ }^{\mathrm{TM}}$ ) that allows varying the full-width at half maximum (FWHM), the attenuation and the central frequency of the filter. The remaining part of the self-tuning cavity consists of the distribution fibre, here of about $10 \mathrm{~m}$ of SSMF and $5 \mathrm{~km}$ of NZDSF. Finally, the gain medium and modulator is constituted by a RSOA designed and realized by Alcatel-Thales III-V Lab [8], whose gain spectrum has a peak centred at $195.775 \mathrm{THz}$. The RSOA in the linear regime presents a low PDG, lower than $1 \mathrm{~dB}$. The receiver used for the BER measurements is an APD-TIA for $1.25 \mathrm{~Gb} / \mathrm{s}$ followed by a clock and data recovery module.

\section{EXPERIMENTAL RESULTS}

The measurement of the cavity optical modes distance is carried out with an out-of coherence interferometer, followed by a PIN with 13-GHz bandwidth and an electrical spectrum analyzer (ESA). In the case of short and long cavity, modes are respectively spaced by nearly $21 \mathrm{kHz}$ and $9 \mathrm{MHz}$, corresponding roughly to the expected cavity length of $22 \mathrm{~m}$ and $9.6 \mathrm{~km}$.

The transmitter output power as a function of the RSOA bias current $\left(I_{\text {bias }}\right)$ has been measured. This has been done for three different FWHMs of the cavity filter $(27 \mathrm{GHz}, 55 \mathrm{GHz}, 110 \mathrm{GHz})$, corresponding to the typical AWG-channel bandwidths with spacing of $50 \mathrm{GHz}, 100 \mathrm{GHz}$ and $200 \mathrm{GHz}$ respectively and for three spectral location of the filter, that is, for filter peak corresponding to the RSOA gain peak $(195.775 \mathrm{THz})$ and shifted of $\pm 400 \mathrm{GHz}$ with respect to the gain peak.
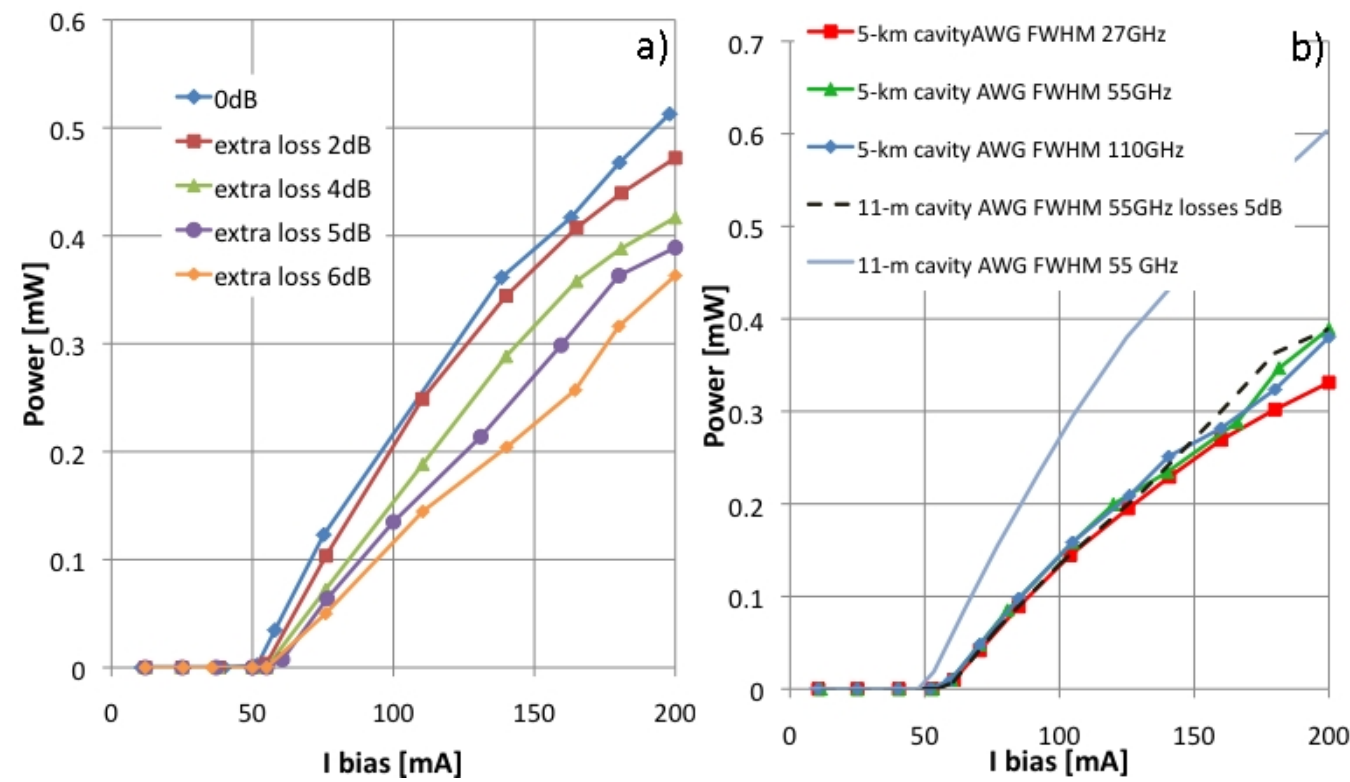

Figure 2. Power versus RSOA bias current for: (a) short cavity length and as function of extra losses in the cavity and (b) long cavity and as a function of AWG FWHMs.

For a given length of the cavity, the PI curves obtained for the three FWHMs and the three positions of the centre frequency of the filter are very similar, confirming that output power is mainly influenced by the overall losses of the cavity independently of AWG bandwidth. Fig 2a) shows the PI curves of the short cavity with the filter at $55 \mathrm{GHz}$ FWHM, centred on the RSOA gain peak with no additional loss (blue squares) and with extra losses up to $6 \mathrm{~dB}$. Figure 2b) presents the PI curves for the $5 \mathrm{~km}$-long cavity and with the three FWHMs. It is interesting to notice that these 5-km curves, which are all superimposed, are very similar to the $11-\mathrm{m}$ curve with $5-\mathrm{dB}$ extra losses (black dashed line). This is consistent with the fact that the 5-km cavity losses are $15.4 \mathrm{~dB}$ that is almost $5 \mathrm{~dB}$ more than the $10.7 \mathrm{~dB}$ of the $11-\mathrm{m}$ cavity losses.

Concerning the BER measurements, two operating points of the cavity have been identified: a lower bias current and lower data voltage applied and a higher bias current and higher data voltage applied. Again the BER measurements at $1.25 \mathrm{~Gb} / \mathrm{s}$ versus the received power have been performed for the various FWHMs and for the three positions of the filter center frequency for both cavity lengths. The best performance is obtained in cases of higher bias current and higher data voltage applied, corresponding to higher extinction ratios (ER). Figure 3a) shows the results for the short cavity as a function of the cavity losses: with increasing losses the penalty 
increases as well. For each curve the corresponding bias current and electrical data amplitude is included. As expected, to maintain cancellation efficiency [9], while cavity losses increase, two strategies can be followed. The data voltages, i.e. ER, can be reduced, yet reducing the eye opening and consequently the performance, as can be seen for instance by comparing the brown triangles (2-dB extra losses) with red circles curve (no extra losses). Alternatively bias current can be increased to recover the extra losses maintaining the applied data voltages, thus only slightly worsening the eye opening, as can be seen by comparing the red-circles curve (no extra losses) with orange squares (4-dB extra losses and lower bias current) and green squares (4-dB extra losses and higher bias current).

Figure $3 b$ ) and 3c) present the analysis carried out varying the filter FWHM, respectively for the short and the long cavity. The worst performance, for both cavity lengths, is obtained with the narrowest filter (27-GHz FWHM), due to the higher associated intensity noise [10]. The cavities with 55-GHz and 110-GHz FWHM filter on the contrary show similar performance. Figure 3c) reports, as dashed lines blue and purple respectively, the performance of the short cavity with $55 \mathrm{GHz}$ FWHM filter and 4 and $6 \mathrm{~dB}$ of extra losses for comparison. The blue-triangles curve, corresponding to the $5-\mathrm{km}$ cavity with the 55-GHz FWHM, is placed between the two dashed curves, as expected as the losses measured in the two cavities differ by about $5 \mathrm{~dB}$. Measurements performed at different filter centre frequencies, not shown in this work, demonstrate less than 0.5 -dB penalty for both working points of the two cavity lengths.
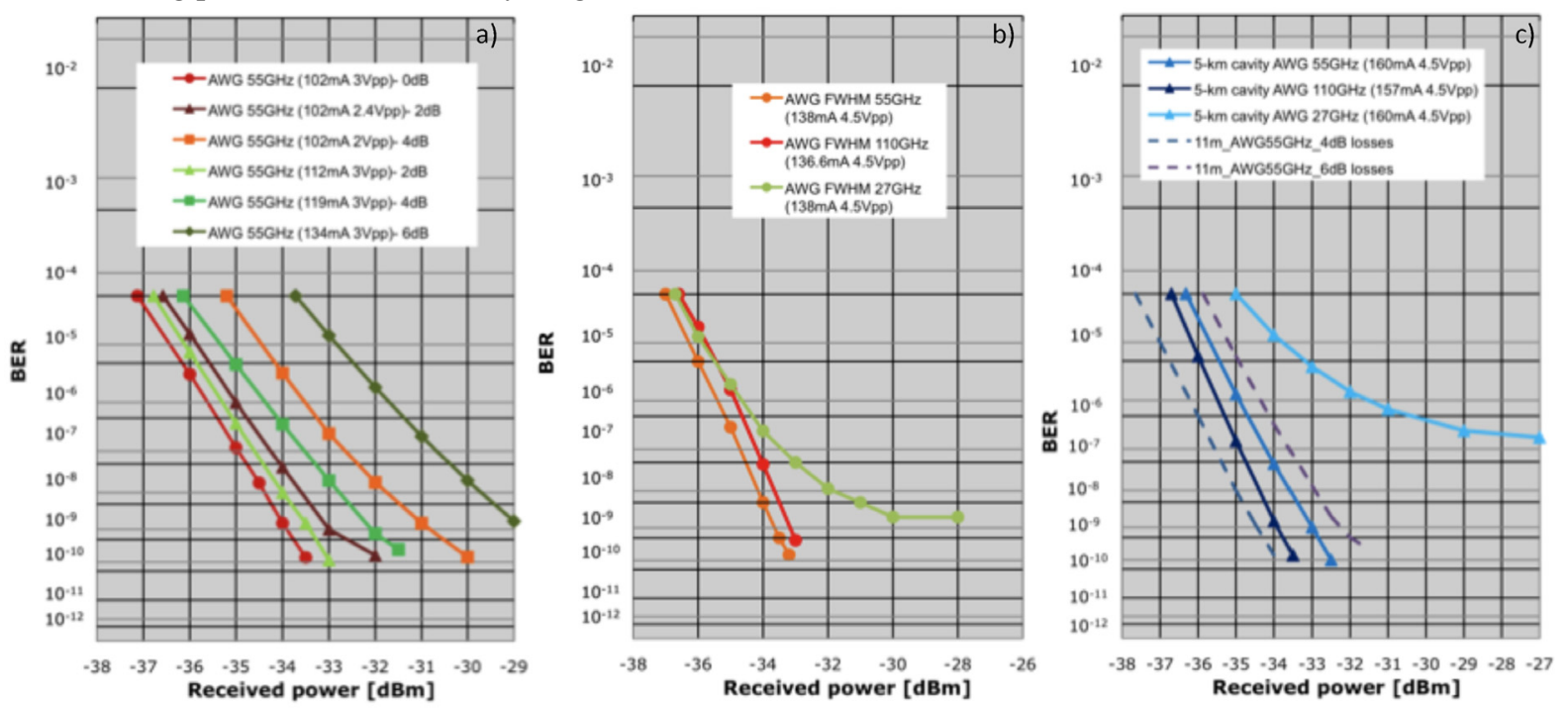

Figure 3. Three BER curves at $1.25 \mathrm{~Gb} / \mathrm{s}$ versus received power: a) short cavity length, low $\mathrm{I}_{\text {bias }}$ 55-GHz filter FWHM, parameter varied: cavity losses; b) short cavity, high $I_{\text {bias, }}$ parameter varied filter FWHM c) long cavity, high $I_{\text {bias }}$, parameter varied filter FWHM.

\section{CONCLUSIONS}

$1.25 \mathrm{~Gb} / \mathrm{s}$ operation of a self-tuning network-embedded transmitter has been analyzed, while varying some parameters characterizing the cavity such as losses, filter FWHMs and filter location with respect to the RSOA gain peak. In particular concerning the optical filter, while its spectral location within the RSOA gain bandwidth is not primarily significant, the filter FWHM determines the transmitter performance as reduced bandwidth (e.g. $27 \mathrm{GHz}$ ) is associated to increased noise levels. Finally the performance is influenced by the cavity losses, which determine the trade-off between eye opening and RSOA capability to cancel recirculating modulation.

\section{ACKNOWLEDGEMENTS}

The authors would like to acknowledge Romain Brenot from Alcatel-Thales III-V Lab for useful discussions and supplying the RSOAs.

This work has been supported by the EU Seventh Framework Programme (FP7/2007-2013) under grant agreement ERMES n ${ }^{\circ} 288542$.

\section{REFERENCES}

[1] C.-H. Lee, et al:: WD-PON experiences in Korea [Invited], J. Opt. Netw. vol. 6, pp. 451-464, 2007.

[2] N. Kashima: Dynamic properties of FP-LD transmitters using side mode injection locking for LAN's and WDM-PONs, J. Lightwave Technol., vol. 24, pp. 3045-3058, Aug. 2006.

[3] S. J. Park, et al.: An evolution scenario of a broadband access network using R-SOA-based WDM-PON technologies, J. Lightwave Technol., vol. 25, pp. 3479-3487, Nov. 2007. 
[4] H. D. Kim, et al.: A low-cost WDM source with an ASE injected Fabry-Perot semiconductor laser, IEEE Photon. Technol. Lett. vol. 12, pp. 1067-1069, Aug. 2000.

[5] G. Talli, et al.: Rayleigh backscattering impairments in access networks with centralised light source, Electron. Lett., 42, 877-878, 2006.

[6] E. Wong, et al.: Directly modulated self-seeding reflective semiconductor optical amplifiers as colorless transmitters in wavelength division multiplexed passive optical networks, J. Lightwave Technol. 25, 67-74, 2007.

[7] M. Presi, et al.: Stable Self-seeding of R-SOAs for WDM-PONs, in Proc. of OFC 2011, paper OMP4.

[8] G. de Valicourt, et al:: High gain $(30 \mathrm{~dB})$ and high saturation power $(11 \mathrm{dBm})$ RSOA devices as colourless ONU sources in long reach hybrid WDM/TDM -PON architecture, IEEE Photon. Technol. Lett. vol. 22, pp. 191-193, Feb. 2010.

[9] L. Marazzi, et al.: Network-embedded self-tuning cavity for WDM-PON transmitter, Opt. Express vol. 20, 3781-3786 (2012).

[10] P. Healey, et al: Spectral slicing WDM-PON using wavelength-seeded reflective SOAs, Electron. Lett., vol. 37, pp. 1181-1182, Sept 2001. 